\title{
CLAIMED, a visual and scalable component library for Trusted Al
}

\author{
Romeo Kienzler ${ }^{* *}$, Ivan Nesic ${ }^{\S}$
}

\begin{abstract}
CLAIMED is a component library for artificial intelligence, machine learning, "extract, transform, load" processes and data science. The goal is to enable low-code/no-code rapid prototyping by providing ready-made components for various business domains, supporting various computer languages, working on various data flow editors and running on diverse execution engines. To demonstrate its utility, we constructed a workflow composed exclusively of CLAIMED components. For this purpose, we made use of a publicly available Computed Tomography (CT) scans dataset [covidata] and created a deep learning model, which is supposed to classify exams as either COVID-19 positive or negative. The pipeline was built with Elyra's Pipeline Visual Editor, with support for local, Airflow and Kubeflow execution.
\end{abstract}

Index Terms-Kubernetes, Kubeflow, JupyterLab, Elyra, KFServing, TrustedAI, Al Explainability, Al Fairness, Al Adversarial Robustness

\section{Introduction}

In our Hospital Research Department we regularly have Citizen Data Scientists (CDS) [citizends] (in our case, mostly medical doctors) working on large corpora of clinical data. Often, monolithic scripts are used for prototyping, lacking quality and reproducibility. Therefore, in cooperation with CDS we've defined the following requirements for a new way of applying data-driven clinical research:

- low-code / no-code environment for rapid prototyping with visual editing and jupyter notebooks

- $\quad$ seamless scaling during development and deployment

- GPU support

- pre-build components for various business domains

- $\quad$ support for the complete python and R tooling including Apache Spark, TensorFlow, PyTorch, pandas and scikitlearn

- seamless extensibility

- reproducibility of work

- data lineage

- collaboration support

We've evaluated the following software tools but we found that these tools, even when used in conjunction, support only a subset of our requirements: Slurm [slurm], Snakemake [snakemake],

* Corresponding author: romeo.kienzler@ch.ibm.com

+ IBM, Center for Open Source Data and AI Technologies (CODAIT)

$\S$ University Hospital of Basel

Copyright $(2021$ Romeo Kienzler et al. This is an open-access article distributed under the terms of the Creative Commons Attribution License, which permits unrestricted use, distribution, and reproduction in any medium, provided the original author and source are credited.
QSub [qsub], HTCondor [htcondor], Apache Nifi [nifi], NodeRED [nodered], KNIME [knime], Galaxy [galaxy], Reana [reana], WEKA [weka], Rabix [rabix], Nextflow [nextflow], OpenWDL [openwdl], CWL [cwl] or Cromwell [cromwell].

To not reinvent the wheel but rather fill the gap, we have built an extensible component library to be used in low-code / no-code environments called CLAIMED - the visual Component Library for Artificial Inteligence (AI), Machine Learning (ML), Extract, Transform, Load (ETL) and Data Science. In the following section we elaborate on the implementation details followed by a description of an exemplary pipeline to showcase the capabilities of CLAIMED. We continue to elaborate on different ideas how CLAIMED can be improved in the "Future Work" section, finally followed by the conclusion.

\section{Implementation}

Before we address how CLAIMED fulfills the previously defined requirements and how the exemplary workflow has been constructed, we will introduce some terms and technologies.

\section{Technology breakdown}

Containerization and Kubernetes: Virtualization opened up a lot of potential for managing the infrastructure, mainly the ability to run different operating systems on the same hardware at the same time. Next step of isolation can be performed for each of the microservices running on the server, but instead of managing access rights and resources on the host operating system, we can containerize these in separate packages with their own environments. Practical effect of this is that we are running each of the microservices as if they have their own dedicated virtual machine, but without the overhead of such endeavour. This is accomplished by running containers on top of the host operating system. An example of the containerization platform is Docker.

With the opportunity to run a vast number of containers, arose the need of their orchestration. The system needs to be constantly monitored and adjusted so that it stays in a desired state. Containers need to be scaled up and down, the communication has to be managed, they have to be scheduled, authentication needs to be managed, there is the need for load balancing etc. There are multiple optins on the market, but Kubernetes is the market leader in this domain. It was donated to Cloud Native Computing Foundation (CNCF) [cncf] by Google, which means a lot of Google's know-how and years of experience went into it. The system can run on public, on-premises or on hybrid clouds. On-premises installation is very important for institutions dealing 
with sensitive data. For IBM, Kubernetes is also strategic. IBM acts as a Kubernetes runtime provider in the cloud and - through the acquisition of RedHat - has become the major vendor for on-premises Kubernetes. IBM is now able to deliver software solutions - so called "Cloud Paks" - on top of Kubernetes, making them run everywhere (hybrid cloud). Therefore, IBM joined CNCF [ibmcncf], and moved all Watson Services to Kubernetes. This makes IBM the 3rd largest committer to Kubernetes. Not only for IBM but also for us, Kubernetes enables the hybrid cloud scenario of transparently moving workload across different onpremises, remote and cloud data centers seamlessly.

Deep Learning with TensorFlow: TensorFlow is the second incarnation of the Google Brain project's scalable distributed training and inference system named DistBelief [tf]. It supports myriad of hardware platforms, from mobile phones to GPU/TPU clusters, for both training and inference. It can even train and run models in browser, without the data ever leaving the user's environment. Apart from being a valuable tool in research domain, it is also being used in demanding production environments. On a development side, representing machine learning algorithms in tree-like structures makes it a good expression interface. Lastly, on the performance vs usability side, both graph and eager modes are supported. Eager mode allows for easier debugging since the code is executed in Python control flow, as opposed to the TensorFlow specific graph control flow [tfeager]. The advantages of graph mode is usage in distributed training, performance optimization and production deployment. In-depth analysis of these two modes can be found here [tfbook].

Kubeflow: Having a compute cluster capable of scaling at container level granularity calls for a workflow execution engine leveraging the advantages of containerization and container orchestration by integrating with Kubernetes seamlessly. This is where Kubeflow [kubeflow] kicks in. It is a machine learning pipeline management and execution system running as first class citizen on top of Kubernetes. Beside making use of Kubernetes scalability, it allows for defining reproducible work products as machine learning pipelines, where results and intermediate artifacts of the executions are stored in a metadata repository.

Jupyter Notebooks / JupyterLab: When it comes to collaboration and reproducibility, document centric coding tools like Apache Zeppelin or Jupyter Notebooks are a great choice. Recently, JupyterLab [jupyter] started setting the standard for the research and data science community [jupyter_standard]. Therefore we consider Jupyter Lab not only as an Integrated Development Environment (IDE) but more as a technology standard practitioners love to work with.

Elyra: Visual editing using drag and drop editing in "no code" / "low code" environments is gaining popularity [lowcode]. As a representative of such environments we introduce Elyra. Elyra [elyra] started as a set of extensions for the JupyterLab ecosystem. Here we concentrate on the pipeline editor, developed by IBM in Open Source under supervision of the authors, which allows for expression of machine learning workflows using a drag and drop editor. Inspired by CWL [cwl] and OpenWDL [openwdl], Elyra uses an open and interchangeable, JSON based format to represent the workflows. This allows Elyra to transpile workflows to different execution engines like Kubeflow or Airflow. This means non-programmers can understand and create machine learning workflows on their own without coding and at the same time making use of Kubernetes massive scalability. Elyra also ships with a browser extension for visualizing such pipelines in the

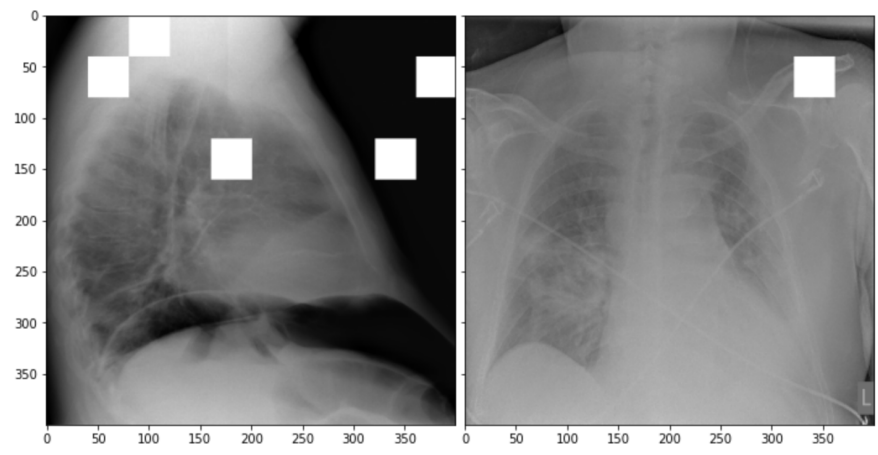

Fig. 1: Example on how LIME helps to identify classification relevant areas of an image.

browser (e.g. from a github repository) to improve collaboration.

AI Explainability: Despite the good performance, deep learning models are viewed as being black box approaches. Technically, deep learning models are a series of non-linear feature space transformations, but an intuitive understanding of each of the individual processing steps is not trivial. There are techniques with which we can look over a deep learning model's shoulder. The one we are using is called LIME [lime]. LIME takes the existing classification model and permutes images taken from the validation set (therefore the real class label is known to LIME) as long as a misclassification is happening. That way LIME can be used to create heat maps as image overlays to indicate regions of images which are most relevant for the classifier. In other words, we identify regions of the image the classifier is looking at.

As Fig. 1 illustrates, the most relevant areas in an image for classifying for COVID-19 are areas containing bones over lung tissue which indicates a problem with that particular classifier.

AI Fairness and Bias: "Bias is a disproportionate weight in favor of or against an idea or thing, usually in a way that is closedminded, prejudicial, or unfair" [bias]. But what we want from our model is to be fair and unbiased towards protected attributes like race, age, socioeconomic status, religion and so on. So wouldn't it be easier if we just "hid" those columns from the model during the training? Unfortunately the problem is convoluted. Protected attributes are often encoded inside the other attributes (latent features). For example, race, religion and socioeconomic status are latently encoded in attributes like zip codes, contact methods or types of products purchased. Therefore, fairness assessment and bias detection is quite challenging. Luckily, a huge number of single number metrics exist to assess bias in data and models. Here, we are using the AIF360 [aif360] library. IBM donated it to the Linux Foundation AI, which puts it under open governance.

AI Adversarial Robustness: Another pillar of Trusted AI is adversarial robustness. For example, as researchers found out, adversarial noise can be introduced in data (data poisoning) or models (model poisoning) to influence models decisions in favor of the adversarial. Libraries like the Adversarial Robustness Toolbox ART [art] support all state-of-the-art attacks and defenses.

\section{Requirements and System Architecture}

In the following section we cover the system architecture and it's requirements. There are two major components: execution engine and integrated tools.

Execution Engine: An execution engine takes a pipeline description and executes it on top of physical machines, reads 


\begin{tabular}{|c|c|c|c|c|c|c|c|}
\hline Requirement & $\mathbf{K F}$ & $\mathbf{A F}$ & Slurm & SM & Qsub & HTCondor & - Reana \\
\hline $\begin{array}{l}\text { Kubernetes Sup- } \\
\text { port }\end{array}$ & $X$ & $\mathrm{X}$ & & $\mathrm{X}$ & & $\mathrm{X}$ & $X$ \\
\hline GPU support & $\mathrm{X}$ & $\mathrm{X}$ & $X$ & $X$ & $\mathrm{X}$ & $\mathrm{X}$ & $X$ \\
\hline $\begin{array}{l}\text { Component } \\
\text { Library }\end{array}$ & $\mathrm{X}$ & & & & & & \\
\hline Reproducibility & $\mathrm{X}$ & $\mathrm{X}$ & & $\mathrm{X}$ & & $\mathrm{X}$ & $X$ \\
\hline Data Lineage & $\mathrm{X}$ & & & & & & $\mathrm{X}$ \\
\hline
\end{tabular}

TABLE 1: Fulfilment of requirements for execution engines. (Abbreviations: $K F=$ Kubeflow, $A F=$ Airflow, $S M=$ Snakemake)

source data and creates output data. The following requirements have been defined in order to assess the adequacy of the execution engine.

- Kubernetes Support

We defined Kubernetes as the lowest layer of abstraction because that way the executor layer is agnostic of the underlying Infrastructure as a service (IaaS) architecture. In addition, Kubernetes provides better resource utilization if multiple pipelines are run in parallel on the system. We can consume Kubernetes as a service (aaS) offered by a variety of Cloud providers like IBM, Amazon, Google, Microsoft, OVH or Linode. A lot of workload for this particular project has been envisioned to be outsourced to SciCore [scicore] - a scientific computing data center part of the Swiss Personalized Health Network (SPHN) [sphn] and the Swiss Institute of Bioinformatics [sib]. Best to our knowledge, their cluster runs on OpenStack and provides Kubernetes as part of it (Magnum). University Hospital of Basel has on-premises RedHat OpenShift platform.

- GPU support

GPU support is essential since a large fraction of the workload is training of deep learning neural networks with TensorFlow and PyTorch. Training those models on CPU doesn't make sense economically and ecologically.

- Component Library

Predefined, ready to use components, are convenient to use, they save time and, if well tested, reduce the probability of an error. Kubeflow for example has components for parallel training of TensorFlow models (TFJob), parallel execution of Apache Spark jobs as a pipeline step, parallel hyperparameter tuning (Katib) and model serving (KFServing/ KNative)

- Reproducibility

From a legal point of view, in certain domains, it is necessary to reconstruct a certain decision, model or output dataset for verification and audit. Therefore the ability to reproduce and re-run a pipeline is a critical requirement. Of course, there are other examples where this is imperative, like in science.

- Data Lineage

Although a subset of reproducibility, Data Lineage is a crucial feature when it comes to visualizing the changes the datasets went through during the pipeline execution.

Integrated tools: Integrated tools are tools which include a visual data flow editor, a component library and an execution engine. Prominent candidates in the open source space are Apache Nifi, NodeRED, KNIME and Galaxy.

\begin{tabular}{|c|c|c|c|c|c|}
\hline Requirement & Nifi & NodeRED & KNIME & Galaxy & Elyra \\
\hline $\begin{array}{l}\text { Kubernetes Sup- } \\
\text { port }\end{array}$ & & & & $\mathrm{X}$ & $X$ \\
\hline GPU support & & & & $\mathrm{X}$ & $\mathrm{X}$ \\
\hline $\begin{array}{l}\text { Component } \\
\text { Library }\end{array}$ & $\mathrm{X}$ & $\mathrm{X}$ & X & $\mathrm{X}$ & $X$ \\
\hline Reproducibility & $\mathrm{X}$ & & $X$ & $\mathrm{X}$ & $X$ \\
\hline Data Lineage & $\mathrm{X}$ & & & $\mathrm{X}$ & $\mathrm{X}$ \\
\hline Visual Editing & $\mathrm{X}$ & $\mathrm{X}$ & $X$ & $\mathrm{X}$ & $X$ \\
\hline $\begin{array}{l}\text { Jupyter } \\
\text { Notebooks }\end{array}$ & & & & & $\mathrm{X}$ \\
\hline
\end{tabular}

TABLE 2: Fulfilment of requirements for integrated tools.

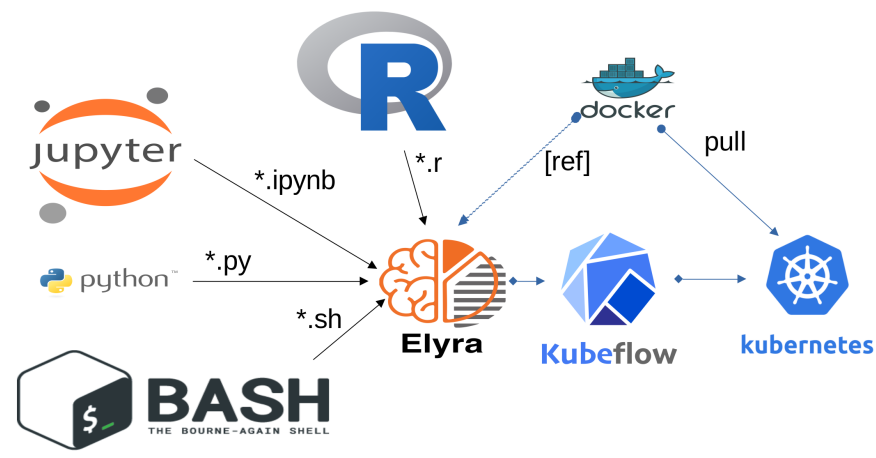

Fig. 2: Runtime architecture of CLAIMED.

The following additional requirements have been defined for a suitable tool:

- Low-Code/No-Code/Visual Editing

Citizen data scientists (in our demo example, medical doctors) need to work with the tool, so visual editing is necessary. But apart from being a visual editing tool, support for creating custom pipeline components on the fly using $\mathrm{R}$ and python is necessary as well.

- Jupyter Notebooks

Researchers in general like to implement tasks in jupyter notebooks. This makes support for JupyterLab, as well as having an easy way of making Jupyter notebooks part of the data processing pipeline, a key requirement.

Final technology choice: As it can be seen from the tables 1 and 2, only Kubeflow on the execution engine side, and Elyra as the integrated tool are capable of covering all of the requirements. Therefore we select this pair as our primary technology choice.

Elyra's pipeline editor supports drag and drop functionality, for adding arbitrary scripts (shell, R, python) and Jupyter notebooks to the canvas. Each script gets a container image assigned to be executed in. At the moment, Elyra supports pipeline submissions to Airflow and Kubeflow.

Together with Kubeflow and JupyterLab (where Elyra runs as an extension), all our requirements are fulfilled.

As it can be seen on Figure 2, Elyra - specifically the pipeline editor of the Elyra Extension to JupyterLab - allows for visually building data pipelines with a set of assets like notebooks and scripts dragged onto a canvas and transparently published to Kubeflow, as a Kubeflow pipeline.

The only thing missing is a set of re-usable notebooks for different kinds of tasks and this is where CLAIMED comes in. 


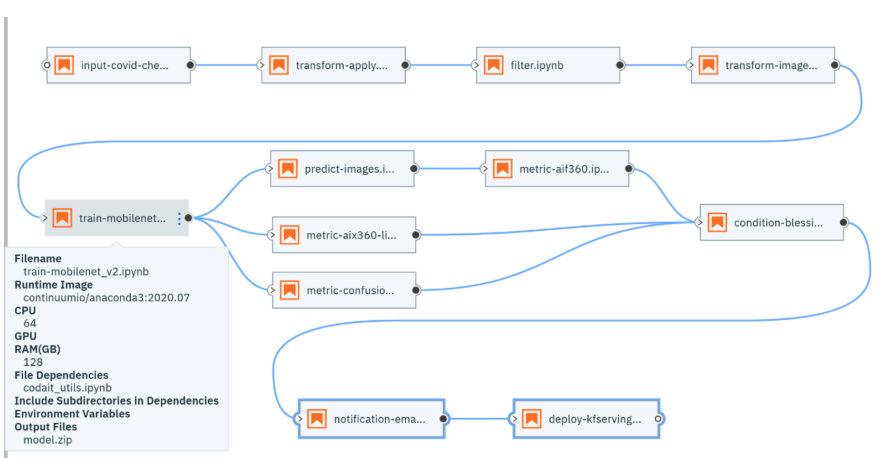

Fig. 3: The exemplary TrustedAI pipeline for the health care use case.

We've published CLAIMED as an open source library [complib]. In the next sections we will introduce the demo use case, along with how components found in CLAIMED have been used to implement this pipeline.

\section{System Implementation and Demo Use Case}

\section{A TrustedAl image classification pipeline}

As mentioned, pipelines are a great way to introduce reproducibility, scaling, auditability and collaboration in machine learning. Pipelines are often a central part of a MLOps strategy. This holds for TrustedAI pipelines too, since reproducibility and auditability are even more important in this case. Figure 3 illustrates the exemplary TrustedAI pipeline we have built using the component library and Figure 4 is a screenshot taken from Kubeflow displaying the pipeline after finishing it's run.

\section{Pipeline Components}

This section exemplifies each existing category with at least one component which has been used for this particular pipeline. There are also other components that are not part of the pipeline, so they are not introduced here. Please note that the core feature of our software is threefold:

- the CLAIMED component library

- Elyra with it's capability to use CLAIMED to create a pipeline and push it to Kubeflow

- the pipeline itself

Input Components: There are input components for different types of data source, like files and databases.

In this particular case, we're pulling data directly from the GitHub repository via a public and permanent link [covidata]. We only pull the metadata.csv and images directory.

Transform Components: Sometimes, transformations on the metadata, or any other structured dataset, are necessary. Therefore, we provide a generic transformation component - in the example, we used it to change to format of the categories as the original file contained forward slashes which made it hard to use on the underlying operating system. This is performed by specifying a column name and a function that has to be applied.

Filter Components: Similar to changing content of rows in a dataset, removing rows is also a common task in data engineering. The filter stage allows doing exactly that. It is enough to provide a predicate - specifically for our case the predicate metadata.filename.str.contains('.gz') removes invalid images.

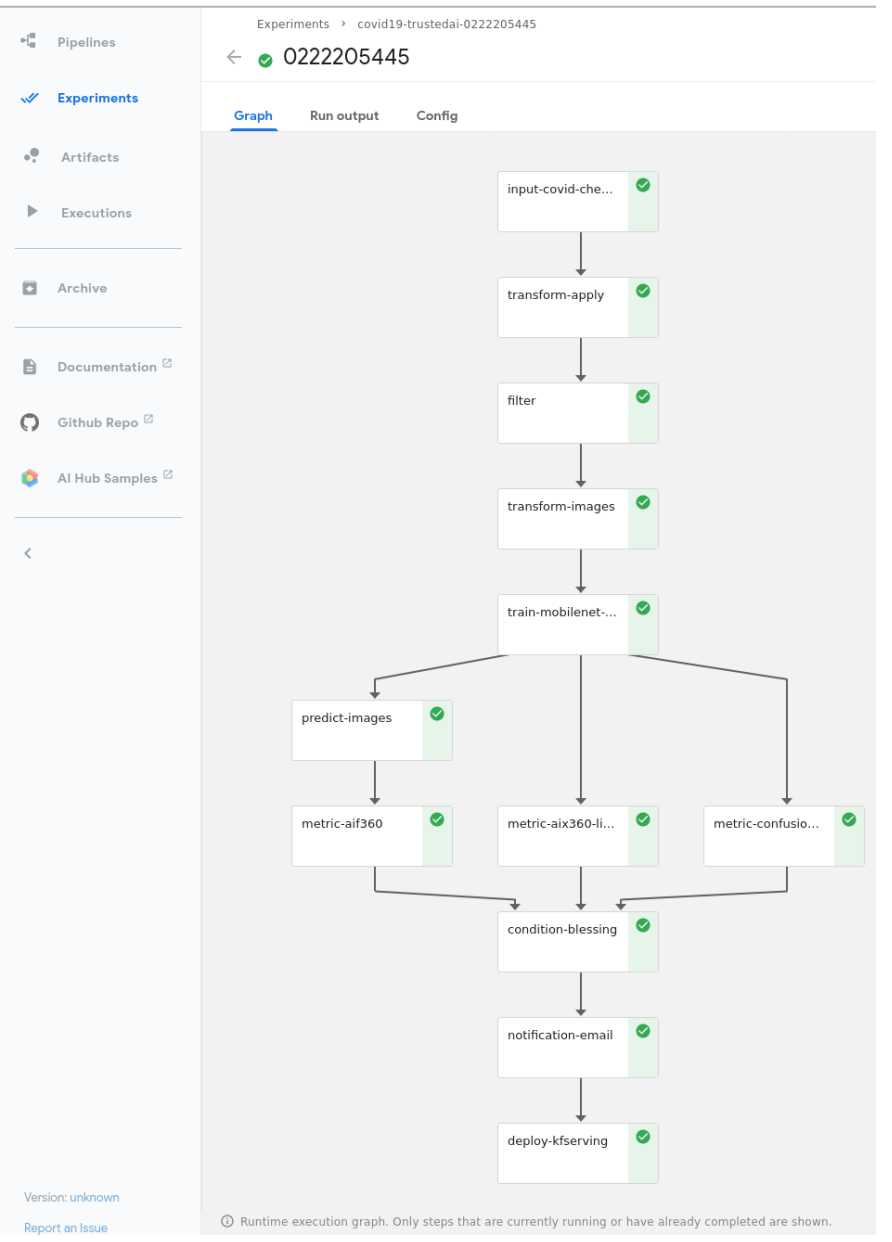

Fig. 4: The pipeline once executed in Kubeflow.

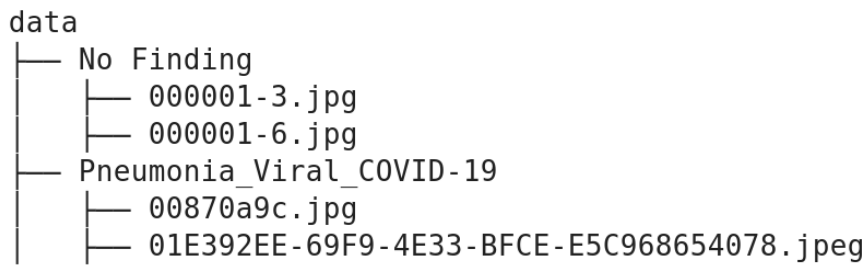

Fig. 5: Example of directory structure supported by TensorFlow Dataset API.

Image Transformer Components: One supported standard for the conversion of image datasets into the TensorFlow's dataset supported format, is to organize images into directories representing their classes [tfimgprep]. TensorFlow Dataset is an API that allows for a convenient way to create datasets from various input data, apply transformations and preprocessing steps and make iteration over the data easier and memory efficient [tfdataset].

In our example, the data isn't in the required format. It is organized as a directory full of images and alongside it is a CSV file which defines the attributes. Available attributes are exam finding, sex and age, from which we only require the finding for our example. The images are then arranged by following the previously described directory structure, as illustrated by Fig. 5. After performing this step, the data can be consumed by the Tensorflow Dataset API. 


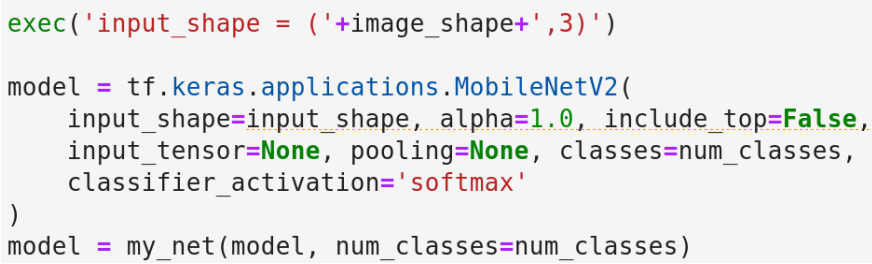

Fig. 6: Source code of the wrapped training component.

Training Components: Understanding, defining and training deep learning models is not a simple task. Training a deep learning image classification model requires a properly designed neural network architecture. Luckily, the community trends towards predefined model architectures, which are parameterized through hyper-parameters. At this stage, we are using the MobileNetV2, a small deep learning neural network architecture with the set of the most common parameters. It ships with the TensorFlow distribution - ready to use, without any further definition of neurons or layers. As shown in Figure 6, only a couple of parameters need to be specified.

Although possible, hyper-parameter search is not considered in this processing stage. The reason being, we want to make use of Kubeflow's hyper-parameter search capabilities leveraged through Katib [katib] in the future.

Evaluation Components: A model needs to be evaluated before it goes into production. Evaluating classification performance against the target labels has been a common metric since the early days of machine learning, therefore we have also developed evaluation components, with confusion matrix support for instance. But taking TrustedAI measures into account is a newly emerging practice. Therefore, components for AI Fairness [aif360], AI Explainability [aix360] and AI Adversarial Robustness [art] have been also added to the component library.

Blessing Components: In Trusted AI (but not limited to) it is important to obtain a blessing of assets like generated data, models or reports to be published and used by other subsystems or humans. Therefore, a blessing component uses the results of the evaluation components to decide if the assets are ready for publishing.

Publishing Components: Depending on the asset type, publishing means either persisting a dataset to a data store, deploying a machine learning model for consumption of other subsystems, or publishing a report to be consumed by humans. Here, we exemplify this category by a KFServing [kfserving] component which publishes the trained TensorFlow deep learning model to Kubernetes. KFServing, on top of KNative, is particularly interesting as it draws from Kubernetes capabilities, like canary deployment and scalability (including scale to zero), in addition to built-in Trusted AI functionality.

\section{Future Work}

We have financial support to add functionality to CLAIMED in multiple dimensions. Below we give a summary of the next steps.

\section{Extend component library}

To this date, at least one representative component for each category has been released. Components are added to the library on a regular basis. The components due to be published are: Parallel Tensorflow Training with TFJob, Parallel Hyperparameter Tuning with Katib and Parallel Data Processing with Apache Spark.

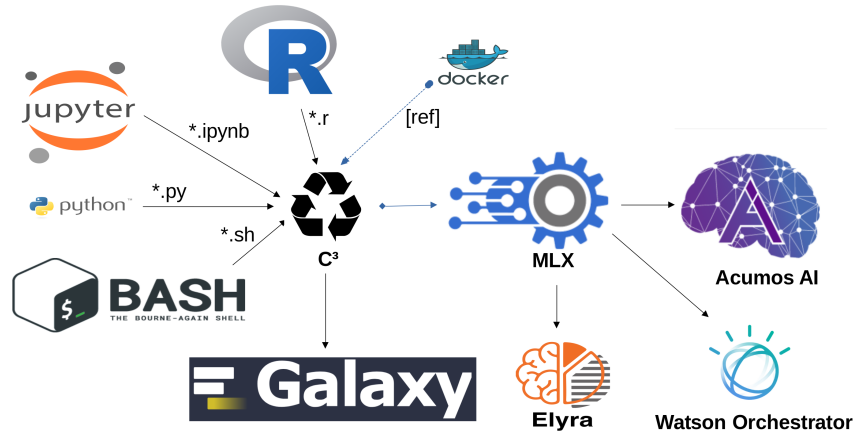

Fig. 7: C3 - The CLAIMED Component Compiler transpiles and publishes pipeline components for different target platforms

\section{Component exporter for Kubeflow}

Containerizing notebooks and scripts is a frequent task in the data science community. In our environment, this involves attaching the arbitrary assets, like jupyter notebooks and scripts, to a container image and then transpiling a Kubeflow component out of it. We are currently in the process of implementing a tool that would facilitate this workflow. The name of the tool is C3 [c3], and it stands for CLAIMED component compiler. Currently, transpiling from notebooks to Kubeflow Pipeline components is supported. In addition, publishing these components to component repositories will also be possible. C3 already supports publishing components to Machine Learning Exchange (MLX) [mlx], an open source asset repository for notebooks, pipelines, data sets, machine learning models and pipeline components. Figure 7 illustrates the concept.

\section{Import/Export of components to/from Galaxy}

As seen in Table 2, Galaxy covers a majority of our requirements already. Unfortunately, Galaxy components - called "tools" are very skewed towards genomics. Adding new components and extending functionality onto other domains would make the tool interesting for a wider audience. Reverse is also true, the existing component library Galaxy is extensive, well established and tested. It makes sense to automatically transpile those tools as components into CLAIMED. We are currently looking into adding import/export support between CLAIMED and Galaxy into C3.

\section{UX improvements of the Elyra pipeline editor}

The components are isolated, so only explicitly shared information can be put into context for all of them. In order for the components' executor, e.g. Kubflow, to do this, it must be provided a configuration. We envision for Elyra to automatically deduce interesting parameters from the code and from the environment, upon which it would create dynamic forms. For example, fields like checkboxes and dropdowns where one can select input and output files mentioned in the code. Currently, only environment variables are provided in a rudimentary UI with one text field per variable. One proposal is to introduce an optional configuration block to the scripts and notebooks. It would then be interpreted by Elyra and the appropriate UI would be rendered.

One successful example of such implementation is Galaxy's UI [galaxy_ui]. A complex UI behavior is expressed by XML configuration. So we are also exploring an option of either using Galaxy's XML Schema or defining a new one and support the transformation from one into the other. 


\section{Add CWL support to the Elyra pipeline editor}

CWL is a powerful workflow expression language supported already by various tools we've evaluated. Currently, Elyra uses its own, proprietary pipeline representation format. Adding support of CWL to Elyra would improve interoperability between different software components. For example, the Reana execution engine used in the particle physics community, and Galaxy (partially) already support CWL. This means it would be possible to export pipelines from Elyra to Reana, without the need of transpiling the pipeline. Alternatively, Elyra could integrate export and import of CWL into its pipeline editor.

\section{Import 3rd party component libraries}

Since the only thing needed for arbitrary code to become a CLAIMED component is to be wrapped in a container image and to be assigned with meta data, it is possible for 3rd party component libraries like those from KNIME or Nifi and to be imported into CLAIMED. This also holds true for Kubeflow components. It is also possible to wrap different components from KNIME, Nifi or similar tools in this manner and use it within Elyra, as well as in the other execution engines CLAIMED supports.

\section{Create more (exemplary) pipelines}

At the moment, CLAIMED ships with three exemplary pipelines. The health care inspired TrustedAI pipeline which was covered in this paper, a pipeline to visualize and predict soil temperature from a historic data set and an IoT sensor data analysis pipeline. The next pipeline in line is a genomics pipeline for the Swiss Institute of Bioinformatics affiliates University Hospital Berne/Berne University and potentially for particle physics at CERN.

\section{Conclusion}

We've build a trustable, low-code, scalable and open source component library, targeting visual data pipeline systems. We've showcased the library's capabilities by building a domain specific pipeline on Elyra, an emerging visual pipeline editor and running it on widely used Kubeflow execution engine. We believe that future import/export functionality of CLAIMED will improve reproducibility of data centric work even further.

\section{REferences}

[art] Maria-Irina Nicolae, Mathieu Sinn, Minh Ngoc Tran, Beat Buesser, Ambrish Rawat, Martin Wistuba, Valentina Zantedeschi, Nathalie Baracaldo, Bryant Chen, Heiko Ludwig, Ian M. Molloy, Ben Edwards Adversarial Robustness Toolbox, arXiv:1807.01069, November 2019

[aif360] Rachel K. E. Bellamy et al. AI Fairness 360: An Extensible Toolkit for Detecting, Understanding, and Mitigating Unwanted Algorithmic Bias, arXiv:1810.01943, October 2018

[aix360] Vijay Arya et al. One Explanation Does Not Fit All: A Toolkit and Taxonomy of AI Explainability Techniques, arXiv:1909.03012, September 2019

[bias] Steinbock, Bonnie (1978). Speciesism and the Idea of Equality, Philosophy, 53 (204): 247-256, doi:10.1017/S0031819100016582

[cncf] Cloud Native Computing Foundation, https://www.cncf.io. Last accessed 18 Feb 2021

[complib] https://github.com/elyra-ai/component-library

[elyra] Elyra AI, https://github.com/elyra-ai. Last accessed $18 \mathrm{Feb}$ 2021 [kubernetes]

[jupyter]

[kfserving]

[lime]

[katib]

[tf]

[ibmencf]

[ect]

[slurm]

[snakemake]

[qsub]

[htcondor]

[galaxy]

[reana]

[nifi]

[nodered]

[knime]

[weka]

[rabix]

[openwdl]

[cwl]
David Bernstein et al. Containers and Cloud: From LXC to Docker to Kubernetes, IEEE Cloud Computing (Volume: 1, Issue: 3), September 2014)

Thomas Kluyver et al. Jupyter Notebooks - a publishing format for reproducible computational workflows, Positioning and Power in Academic Publishing: Players, Agents and Agendas, 87-90, doi:10.3233/978-1-61499. 649-1-87, 2016

Clive Cox and Dan Sun and Ellis Tarn and Animesh Singh and Rakesh Kelkar and David Goodwin, Serverless inferencing on Kubernetes, Workshop on "Challenges in Deploying and Monitoring Machine Learning System" at ICML 2020

Marco Tulio Ribeiro et al. "Why Should I Trust You?": Explaining the Predictions of Any Classifier, Proceedings of the 22nd ACM SIGKDD International Conference on Knowledge Discovery and Data Mining, San Francisco, CA, USA, pp. 1135-1144 (2016), doi:10.1145/2939672.2939778

Debo Dutta and Xinyuan Huang, Consistent Multi-Cloud AI Lifecycle Management with Kubeflow, OpML, 2019

George et al. A Scalable and Cloud-Native Hyperparameter Tuning System, arXiv:2006.02085, June 2020

Martín Abadi et al. TensorFlow: Large-Scale Machine Learning on Heterogeneous Distributed Systems, arXiv: $1603.04467 \mathrm{v} 2$, March 2016

IBM joining CNCF, https://developer.ibm.com/ technologies/containers/blogs/ibms-dedication-to-opensource-and-its-involvement-with-the-cncf Last accessed 18 Feb 2021

https://github.com/cloud-annotations/elyra-classificationtraining/tree/developer_article

"Yoo, Andy B. and Jette, Morris A. and Grondona, Mark, SLURM: Simple Linux Utility for Resource Management, Job Scheduling Strategies for Parallel Processing, Springer, 2003

Köster, Johannes and Rahmann, Sven, Snakemake-a scalable bioinformatics workflow engine, Journal of Bioinformatics, Number 19, Volume 28, Pages 2520-2522\}, August 2012

https://en.wikipedia.org/wiki/Qsub

E M Fajardo et al, How much higher can HTCondor fly?, 2015 Journal of Physics.: Conference Series, Volume 664, June 2014

Enis Afgan et al. The Galaxy platform for accessible, reproducible and collaborative biomedical analyses: 2018 update, Nucleic Acids Research, 46):W537-W544, doi:10.1093/nar/gky379, July 2018

Tibor Šimko et al. REANA: A System for Reusable Research Data Analyses, 23rd International Conference on Computing in High Energy and Nuclear Physics (CHEP 2018), (214):06034, doi:10.1051/epjconf/201921406034, September 2019

https://nifi.apache.org/

Z. Chaczko and R. Braun, Learning data engineering: Creating IoT apps using the node-RED and the RPI technologies, 16th International Conference on Information Technology Based Higher Education and Training (ITHET), 1-8, doi: 10.1109/ITHET.2017.8067827, 2017

Michael R. Berthold et al. KNIME - the Konstanz information miner: version 2.0 and beyond, ACM SIGKDD Explorations Newsletter, (11):26-31, doi:10.1145/1656274.1656280, June 2009

Mark Hall et al. The WEKA data mining software: an update, ACM SIGKDD Explorations Newsletter, (11):10-18, doi:10.1145/1656274.1656278, June 2009

Gaurav Kaushik et al., RABIX: AN OPEN-SOURCE WORKFLOW EXECUTOR SUPPORTING RECOMPUTABILITY AND INTEROPERABILITY OF WORKFLOW DESCRIPTIONS, Proceedings of the Pacific Symposium on Biocomputing 2017, (22):154-165, doi:10.1142/9789813207813_0016, November 2017

Di Tommaso, P., Chatzou, M., Floden, E. et al. Nextflow enables reproducible computational workflows., Nature Biotechnology, (35):316-319, doi:10.1038/nbt.3820, 2017 https://openwdl.org/

https://www.commonwl.org/ 
[cromwell] https://cromwell.readthedocs.io/en/stable/

[covidata] Joseph Paul Cohen et al. COVID-19 Image Dato

Collection: Prospective Predictions Are the Future, arXiv:2006.11988, 2020

[tfeager] Akshay Agrawal et al. TensorFlow Eager: A Multi-Stage, Python-Embedded DSL for Machine Learning, Proceedings of the 2nd SysML Conference, arXiv:1903.01855, 2019

[tfdataset] Steven W. D. Chien et al. Characterizing Deep-Learning I/O Workloads in TensorFlow, IEEE/ACM 3rd International Workshop on Parallel Data Storage - Data Intensive Scalable Computing Systems (PDSW-DISCS), doi:10.1109/PDSW-DISCS.2018.000112018

[tfimgprep] https://www.tensorflow.org/api_docs/python/tf/keras/ preprocessing/image_dataset_from_directory

[galaxy_ui] https://github.com/bgruening/galaxytools/blob/ c1027a3f78bca2fd8a53f076ef718ea5adbf4a8a/tools/ sklearn/pca.xml\#L75

[c3] https://github.com/romeokienzler/c3

[tfbook] Romeo Kienzler and Jerome Nilmeier, What's New In TensorFlow 2.x?, O'Reilly Media, Inc., ISBN: 9781492073710 , July 2020

[vscode] https://code.visualstudio.com/

[scicore] https://scicore.unibas.ch/

[sphn] https://sphn.ch/

[sib] https://www.sib.swiss/

[citizends] Mullarkey, Matthew T. et al., Citizen Data Scientist: A Design Science Research Method for the Conduct of Data Science Projects, Extending the Boundaries of Design Science Theory and Practice, 191-205, Springer International Publishing, ISBN 978-3-030-19504-5, 2019

[jupyter_standard] Perkel, Jeffrey M. Why Jupyter is data scientists' computational notebook of choice. Nature, Volume 563, Number 7732, Page 145+, 2018

[lowcode] Apurvanand Sahay et al. Supporting the understanding and comparison of low-code development platforms, 171-178, IEEE 46th Euromicro Conference on Software Engineering and Advanced Applications (SEAA), doi:10.1109/SEAA51224.2020.00036, 2020

[mlx] https://github.com/machine-learning-exchange/mlx, Accessed: 29 of June, 2021 